\title{
CORRECTION
}

\section{Correction to: Mapping the vestibular cerebellar evoked potential (VsCEP) following air- and bone-conducted vestibular stimulation}

\author{
Sendhil Govender ${ }^{1} \cdot$ Neil P. M. Todd ${ }^{3} \cdot$ James G. Colebatch $^{1,2}(0$
}

Published online: 5 June 2020

๑) Springer-Verlag GmbH Germany, part of Springer Nature 2020

\section{Correction to: \\ Experimental Brain Research (2020) 238:601-620 \\ https://doi.org/10.1007/s00221-020-05733-x}

In the original publication of the article, Fig. 3 was incorrect. The issue relates to the reporting of variance estimates associated with a single panel of Fig. 3. The correct Fig. 3 and values are given below:

The original article can be found online at https://doi.org/10.1007/ s00221-020-05733-x.

James G. Colebatch

j.colebatch@unsw.edu.au

1 Institute of Neurological Sciences, Prince of Wales Hospital, Randwick, Sydney, NSW 2031, Australia

2 Neuroscience Research Australia, University of New South Wales, Randwick, Sydney, NSW 2052, Australia

3 Department of Psychology, University of Exeter, Exeter EX4 4QC, UK 
A

Single subject data

RMS average

Evoked response

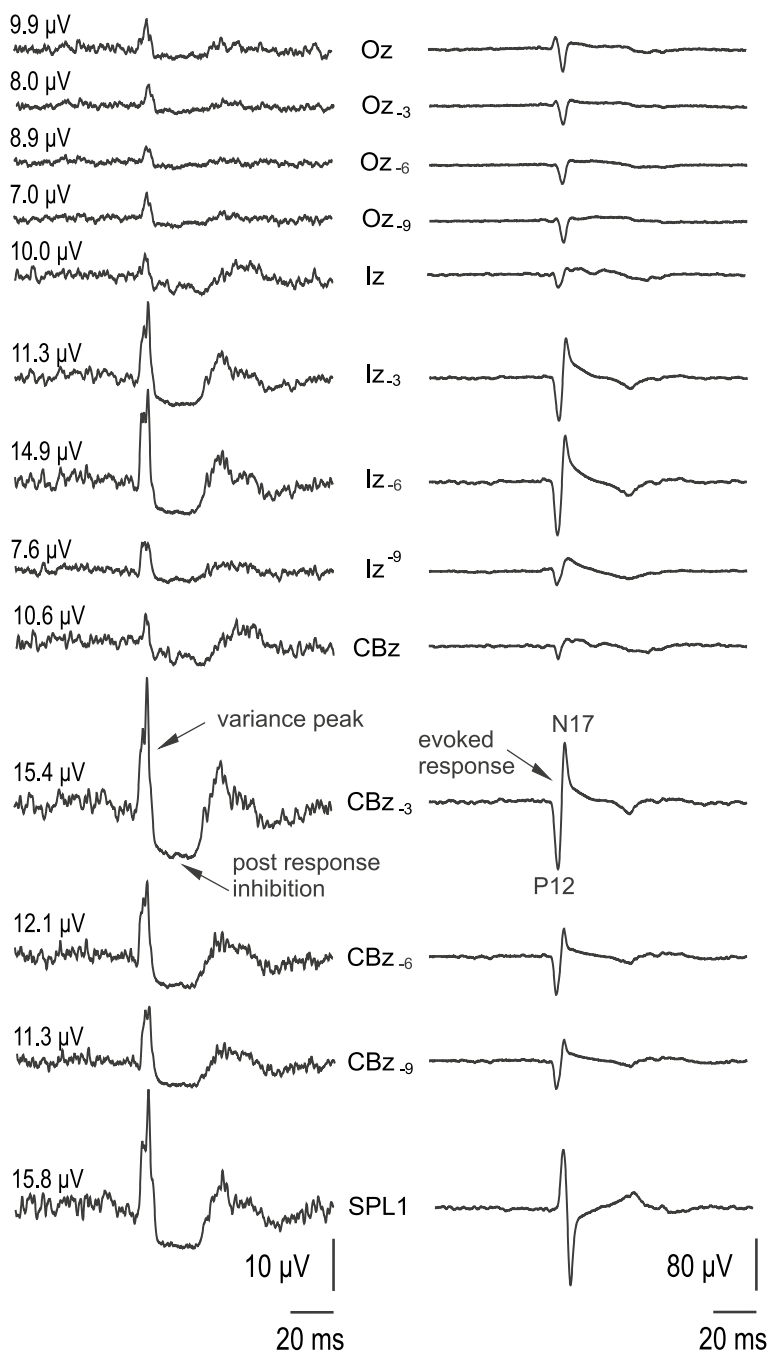

\section{Grand means}

\section{B \\ Correlations}

Inhibition vs evoked response

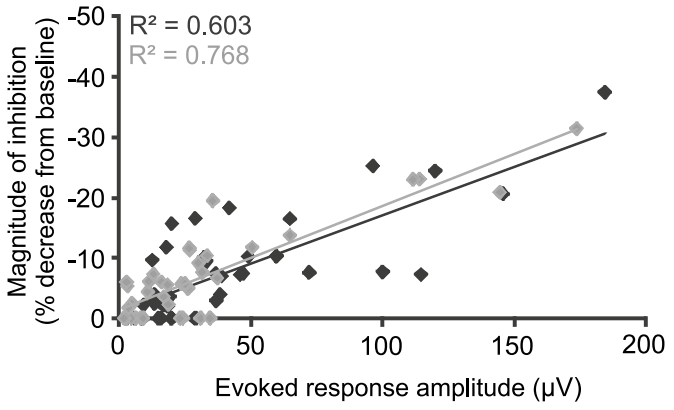

Inhibition (duration) vs evoked response

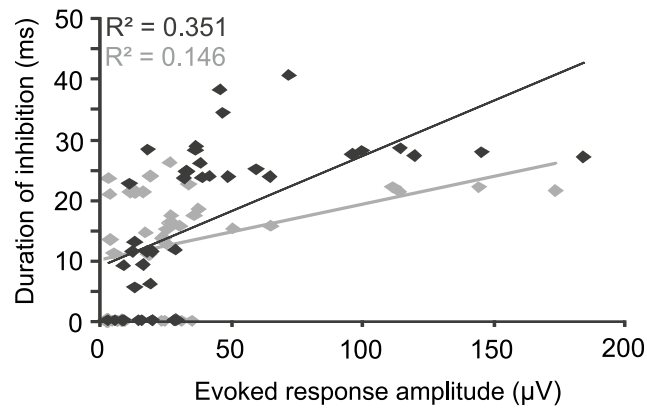

Variance peak vs evoked response

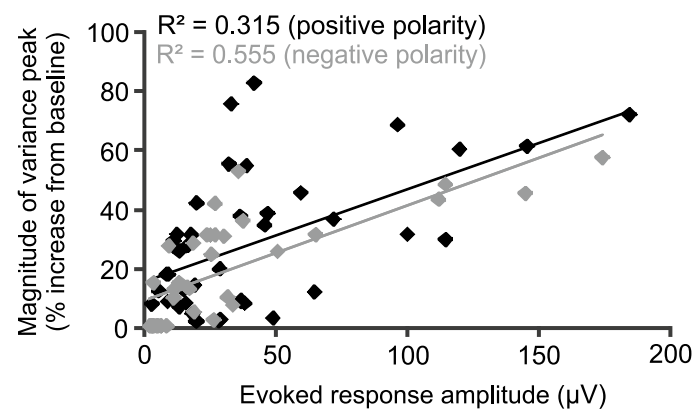

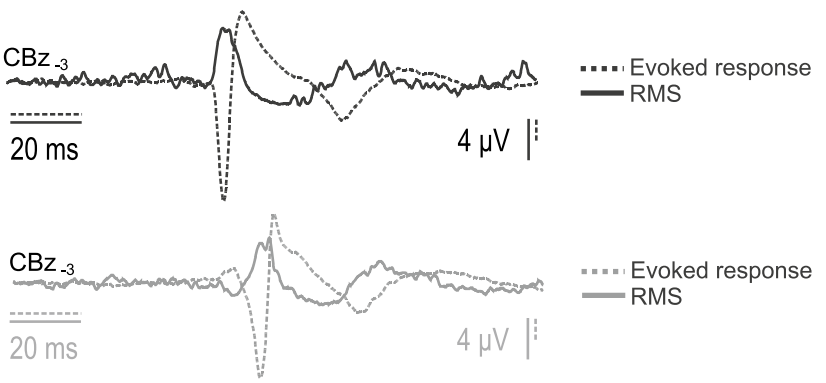


4Fig. 3 a Single subject data (19-year-old male) demonstrating the relationship between the RMS average and the evoked response. The variance peak and post-response inhibition are shown across the array of left-sided electrodes following right mastoid stimulation using positive polarity $\mathrm{BC}$ impulses. The variance peak and periods of inhibition were greater in electrode locations which produced largest evoked responses during mapping. In this subject, a prominent inverted response can be seen in the SPL1 electrode. Values on the RMS averages indicate prestimulus baseline levels which were used to calculate the percentage increase (variance peak) or decrease (postresponse inhibition) in each subject. b Both the magnitude of the variance peak and inhibition demonstrated positive correlations with the size of the evoked response for both positively (black) and negatively (grey) directed BC impulses. The duration of inhibition was strongly correlated with the evoked response for positive polarity BC impulses. c Grand mean traces $(n=10)$ that superimpose the evoked response on the RMS average for the $\mathrm{CBz}_{-3}$ electrode site. Similar averages were also observed for the $\mathrm{Iz}_{-3}, \mathrm{Iz}_{-6}$ and $\mathrm{CBz}_{-6}$ sites. The initial biphasic evoked response corresponded with the variance peak whereas the period of inhibition corresponded to the slow wave decrease following the initial evoked response. Both positive (black) and negative (grey) polarities demonstrated this relationship

The average size of the preceding peak of variance as a $\%$ increase from baseline levels was $28.9 \pm 23.0 \%$ [2.3-82.9\%] for positive polarity $\mathrm{BC}$ impulses and $19.2 \pm 17.8 \%$
[0-57.6\%] for negative polarity BC impulses. For both stimulus polarities, strong positive correlations were shown between the size of the evoked response and the variance peak (P12-N17 response: $r=0.561, \mathrm{P} 20-\mathrm{N} 26$ response: $r=0.744, P<0.001$ for both) (Fig. 3b).

Publisher's Note Springer Nature remains neutral with regard to jurisdictional claims in published maps and institutional affiliations. 